\title{
Identification of analytical factors affecting complex proteomics profiles acquired in a factorial design study with ANOVA - simultaneous component analysis.
}

Vikram Mitra, Natalia Govorukhina, Gooitzen Zwanenburg, Huub Hoefsloot, Inge Westra, Age

Smilde, Theo Reijmers, Ate G. J. van der Zee, Frank Suits, Rainer Bischoff, Péter Horvatovich

\section{Materials and methods}

\subsection{LC-MS/MS (QTOF)}

For the analysis of 40 depleted serum samples, the HPLC equipment and elution program was identical to the LC-MS analyses on the iontrap (see section 1.4.1. in the main manuscript), while MS/MS analysis was performed using a quadrupole time-of-flight mass spectrometer (qTOF, Agilent 6510). Data dependent LC-MS/MS analysis was performed using $2 \mathrm{GHz}$ extended dynamic range mode collection of $3 \mathrm{MS} / \mathrm{MS}$ in one duty cycle under the following additional parameters: mass range: 275-2000 m/z, acquisition duty cycle: 1 spectrum/sec, data storage: profile and centroid mode, fragmentor: $175 \mathrm{~V}$, skimmer: $65 \mathrm{~V}$, OCT 1 RF Vpp: $750 \mathrm{~V}$, spray voltage: $1900 \mathrm{~V}$, drying gas temp: $325^{\circ} \mathrm{C}$, drying gas flow $\left(\mathrm{N}_{2}\right): 6 \mathrm{~L} / \mathrm{min}$. Mass correction was performed during analysis using internal standards of $371.31559 \mathrm{~m} / \mathrm{z}$ (originating from a ubiquitous background ion Dioctyl adipate, DOA, plasticizer) and $1221.990637 \mathrm{~m} / \mathrm{z}$ (HP-1221 calibration standard, evaporating from a wetted wick inside the spray chamber).

\subsection{Data pre-processing and quantification}

Figure 1 shows the workflow that illustrates the steps in the analysis of the experimental design data. Raw iontrap single stage LC-MS datasets were obtained in Bruker Daltonics HPLC-MS.dat format which was further converted into the mzXML proteomics standard format using the msConvert tool from the ProteoWizard toolset ${ }^{1,2}$. The Threshold-Avoiding Proteomics Pipeline (TAPP) ${ }^{3}$ was used to extract chromatographic peaks from the raw data and for data pre-processing. Centroid data were smoothed and reduced using a normalized two dimensional Gaussian filter with a peak resolution in $\mathrm{m} / \mathrm{z}$ dimension of $\sigma_{\mathrm{m} / \mathrm{z}}=0.3 \mathrm{~m} / \mathrm{z}$. This parameter was obtained by optimizing the peak detection quality upon visual inspection of one chromatogram (see Figure S5). Smoothing low-resolution single stage iontrap data with $\sigma_{\mathrm{m} / \mathrm{z}}=0.3 \mathrm{~m} / \mathrm{z}$ and $\sigma_{\mathrm{rt}}=0.5$ minutes using a 2-dimensional Gaussian filter results in one peak without isotopic resolution for each peptide isotope cluster for a given charge state. The nonlinear retention time shifts between LC-MS peak lists were corrected using Warp2D, which is a tool based on Correlation Optimized Warping (COW). To find the best reference chromatogram, all possible pairwise combinations of alignments were performed resulting in a total of 256 pairwise alignments using distributed grid computing with the Data Analysis Framework (DAF) ${ }^{4}$. The parameters for time alignment were as follows: retention time width: 0.5 minutes; m/z width 0.3 Da; maximal retention time difference 0.6 minutes; maximum $\mathrm{m} / \mathrm{z}$ difference $0.6 \mathrm{Da}$; windows size: 50 points; slack parameter: 10 points; maximal number of peaks/segment: 50; number of total time points: 2 000; constant retention time shift: 0 min. The raw data was analysed for peaks with 100-1 $500 \mathrm{~m} / \mathrm{z}$ and 65-135 minutes of retention time. Every alignment combination with Warp2D produced a quality score between 0 (no alignment and/or no peak list similarity) and 1 (perfect alignment and peak list similarity). The peak list with the highest geometrical mean of the sum of overlapping peak volumes normalized to the sum of peak volumes of the two individual chromatograms after warping to all combinations was selected as the optimal reference (sample ID 16090525). All other peak lists were aligned and corrected to this reference and used for further processing. Corresponding peaks in multiple chromatograms were matched with the MetaMatch module of the TAPP pipeline using the following parameters: delta $\mathrm{m} / \mathrm{z}$ : $0.3 \mathrm{Da}$; delta retention time: 0.5 minutes; minimal fraction of class occupancy of peaks: 0.50 , meaning that a matched peak was retained if the peak was identified in a 
minimum of 8 out of the 16 analysed samples. Isolated peaks that did not belong to a peak cluster were removed as "orphaned" peaks. Finally a quantitative peak matrix containing the intensity, average $\mathrm{m} / \mathrm{z}$ ratio and average retention time information of 2559 common peaks was obtained and used in the following statistical analysis. The resulting quantitative peak matrix contained 13,492 zeros, corresponding to $32.95 \%$ of the total number of peaks. The intensity of the orphaned peaks was considered as representative of the noise level. Gaussian mixture curves were fitted to the natural logarithm of the intensity of the orphaned peaks. This analysis resulted in a normal distribution for the noise, $N(\mu=6.042, \sigma=0.5391)$. This distribution was used to noise-fill the zeros in the peak matrix.

\subsection{Annotation of the aligned peak matrix}

All depleted human serum samples for the experimental design were analysed in single stage (MS ${ }^{1}$ ) mode, therefore no identification of peaks with MS/MS spectra was possible. In order to annotate the quantitative peak matrix obtained with the TAPP pipeline, we used the data of 40 depleted human serum samples obtained with the same sample preparation and analysed by a QTOF instrument using the same LC columns and elution conditions. The obtained LC-MS/MS data was identified with the PEAKS 7.5 database search tool ${ }^{5}$ with the following parameters: database: Uniprot (July 22, 2015); parent mass error tolerance: $50.0 \mathrm{ppm}$; fragment mass error tolerance: $0.05 \mathrm{Da}$; precursor mass search type: monoisotopic; enzyme: trypsin; max missed cleavages: 2; non-specific cleavage: one variable modifications: Oxidation (M); max variable ptm per peptide: 3; searched entry: 29,5778; MS/MS quality filter: >0.65; FDR (Peptide-Spectrum Matches): 0.1\%; FDR (Peptide Sequences): 1.0\%; FDR (Protein): $0.0 \%$ determined with reverse decoy approach were retained and used for annotation transfer. The dataset contained 356,633 MS/MS scans, and the database search resulted in 17,4331 peptidespectrum matches, 1884 identified unique peptide sequences, 2,417 unique peptides of different charge and oxidation states, 106 protein groups and 229 identified proteins. The FDR rate for PSM, peptides and proteins were $<1 \%$. The iontrap data was identified using the same parameters except for the following: parent mass error tolerance: $0.5 \mathrm{Da}$; fragment mass error tolerance: $0.3 \mathrm{Da}$. The search for iontrap resulted in 334 peptide-spectrum matches, 163 unique peptide sequences, 183 unique peptides of different charge and oxidation states, 33 protein groups and 113 proteins. Since the iontrap LC-MS data used for quantification was obtained with a different instrument than the QTOF LC-MS/MS data used for peptide identification it was necessary to check if the elution order of common peaks in the two analysis batches is the same ${ }^{6}$. Two depleted human serum samples (sample IDs 30B and 29B) were analysed in QTOF and in the iontrap using MS/MS mode. A database search of these two analyses resulted in 181 unique peptides of different charge and oxidation states identified in both Q-TOF and iontrap datasets. The analysis of two different samples with QTOF and iontrap instruments separately resulted in 470 and 27 MS/MS with common identification respectively (plots 3 and 1 in Figure S6 in supporting information). Due to the low number of identified common peptides it was not possible to apply our quality control method assessing peak elution order inversion ${ }^{6}$, however visual inspection of the scatter plot of the MS/MS spectra and the calculated $\mathrm{D}_{\max }$ (Figure S6 in supporting information) showed slight orthogonality of the separation despite the fact that the same liquid chromatography system, elution condition and column were used ${ }^{7}$. This setup allows to transfer the peptide identifications from the 40 QTOF MS/MS files with a minimal error of 2 minutes as determined by $D_{\max }$ to annotate the peaks in the single stage MS profile of the experimental design dataset, however, the results should considered with care. The scheme of the main steps of identification transfer is presented in Figure S7 (supporting information). Using the derived non-linear retention time correspondence function the retention times of 2,417 unique peptides with different charge and oxidation states in the QTOF datasets were aligned to the iontrap LC-MS dataset. The corrected retention time coordinates of QTOF's unique peptides were used to annotate peaks corresponding to peptides in the pre-processed iontrap experimental design LC-MS dataset. In the annotation we allowed $0.85 \mathrm{~m} / \mathrm{z}$ and $3.5 \mathrm{~min}$ of retention time difference between $\mathrm{m} / \mathrm{z}$ and the retention time of peptide identifications and peak position in the matched single stage experimental design dataset. The matching procedure resulted in 629 peaks annotated with peptide and protein identification. For the annotation of peaks most affected by significant factors in Figure $\mathbf{3}$ and Table $\mathbf{S 2}$ we have used protein names corresponding to SwissProt identifiers. 


\subsection{Parameters of the simulated dataset}

We have simulated data matrix $\mathrm{X}$ with the same dimensions as the data matrix $\mathrm{X}$ obtained from the experimental design LC-MS dataset with goal to assess ASCA performance to identify significant preanalytical factor, to identify peaks affected by significant factors and to assess ASCA performance with respect of peak selection using Volcano plot parameters (t-test p-value and fold change).

The $\mathbf{X}$ matrix of dimension $(2,559 \times 16)$ was obtained as follow: from the seven pre-analytical factors, three (factors 1, 3 and 5) were constructed to have a significant effect on 5\%, 3.5\% and 5\% of randomly chosen peaks with a mean difference in peak intensity between the two factor levels of 3,4 and 6, respectively. Any peaks not affected by factors was sampled from noise distribution found in the experiment design data and using normal distribution of $\mathrm{N}(\mu=6.042, \sigma=0.539)$. The peak intensities obtained with this approach for the seven factors were averaged out and the outcome was used as simulated data matrix $\mathbf{X}$ for ASCA analysis.

To test eventual overfitting we have simulated completely random data matrix $\mathbf{X} 15$ times with the same dimensions than the experimental design dataset, where all factors were non-significant and there was no peak affected by any of the factors. During the simulation we have analysed the complete simulated data matrix and matrix obtained after Volcano based filtering using the same set of threshold used during assessment of ASCA performance with simulation. Figures from these analysis are available in file ParameterOptFactdesRandom.pdf submitted as supporting information.

\subsection{Main steps requiring bioinformatics intervention}

Three steps require bioinformatics intervention: 1.) planning experimental design providing level distribution of the different factors can be performed with MODDE software; 2.) data pre-processing of LC-MS(/MS) resulting in a table that contains quantitative information on compounds for all samples designed in point 1 , which data preprocessing can be performed with any single-stage LC-MS(/MS) processing workflow such as TAPP ${ }^{3}$, OpenMS ${ }^{6}$, mzMine $^{7}$ or maxQuant ${ }^{8}$ and 3.) the ASCA analysis (matlab script provided at https://github.com/vikrammitra/ASCA). 


\section{Supporting figures}

\section{Simulated dataset}

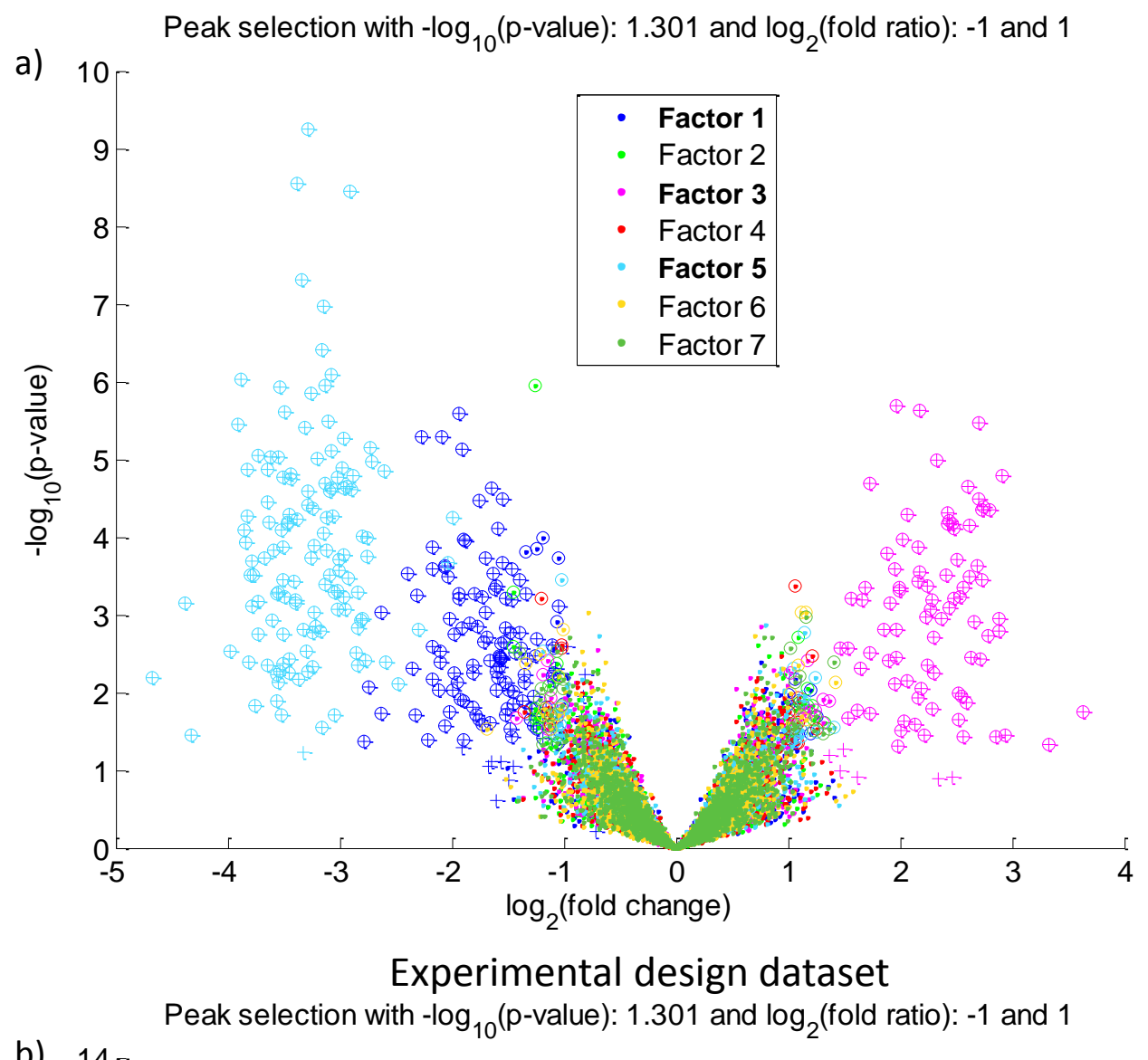

b) 14

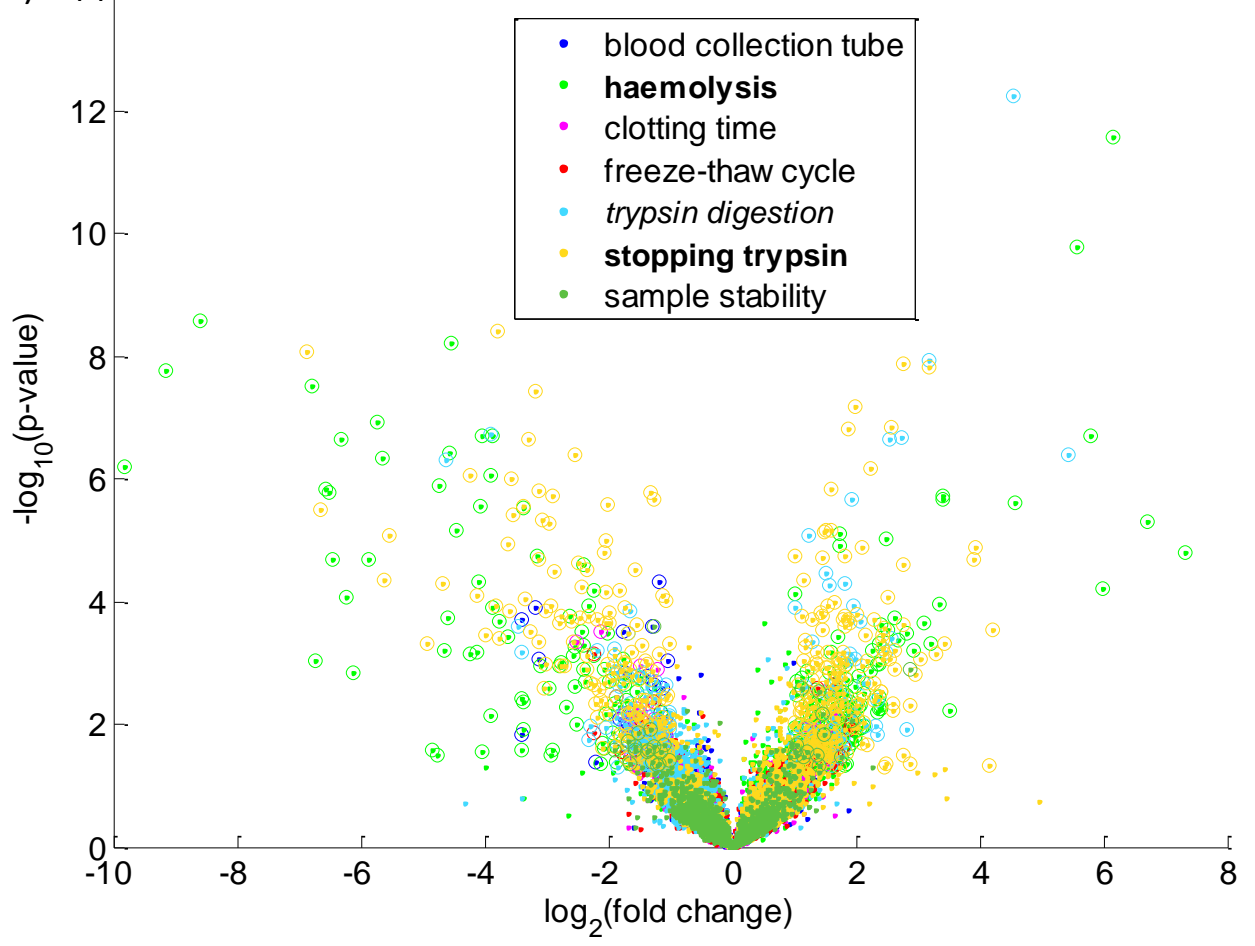

Figure S1. Volcano plots of the matched peak matrices using the low and high levels of seven factors leading to $7 \times 2,559$ dots. a) simulated dataset, b) experimental design dataset. In the simulated dataset peaks sampled with different means between the high and low level of factors 1, 3 and 5 are shown with + , while all other peaks are represented by dots. The factors are represented by differently colored symbols. Peaks selected for ASCA analysis using a threshold of 2 and 0.05 for fold ratio change and $t$ test significance, respectively, are encircled. 

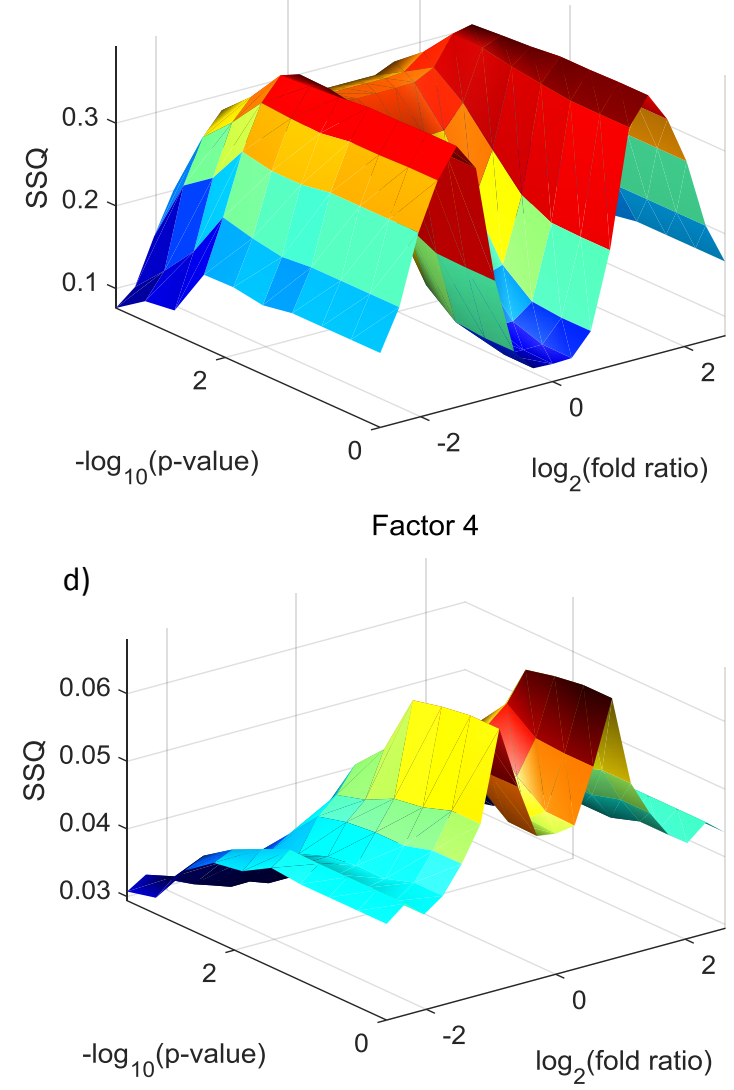

b)

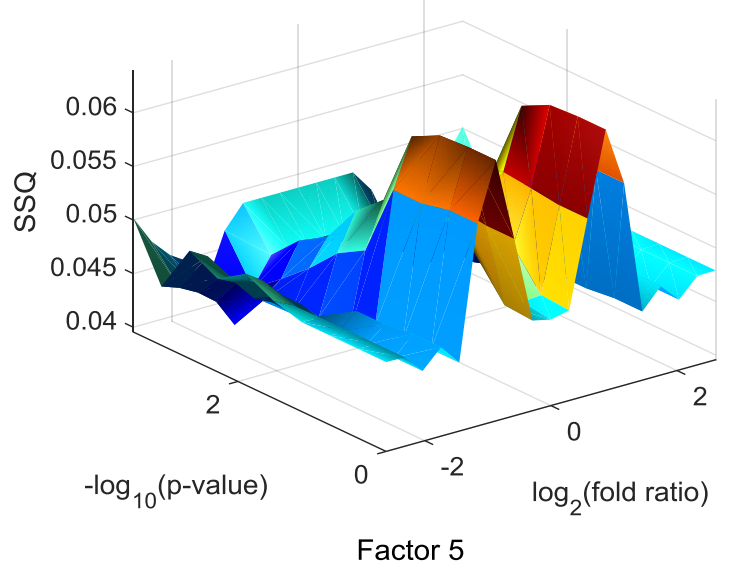

e)

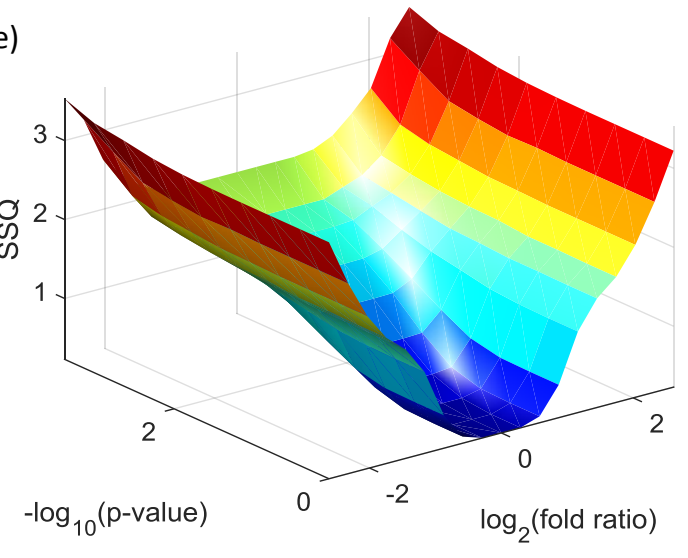

Factor 7

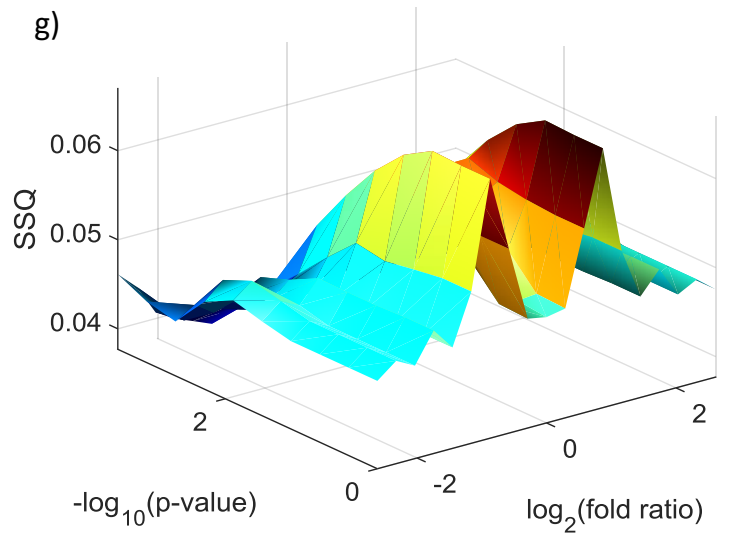

c) Factor 3

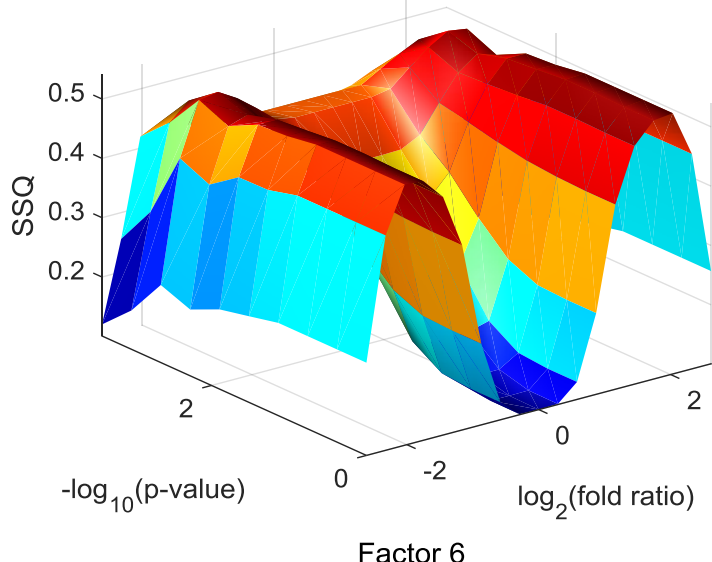

f)

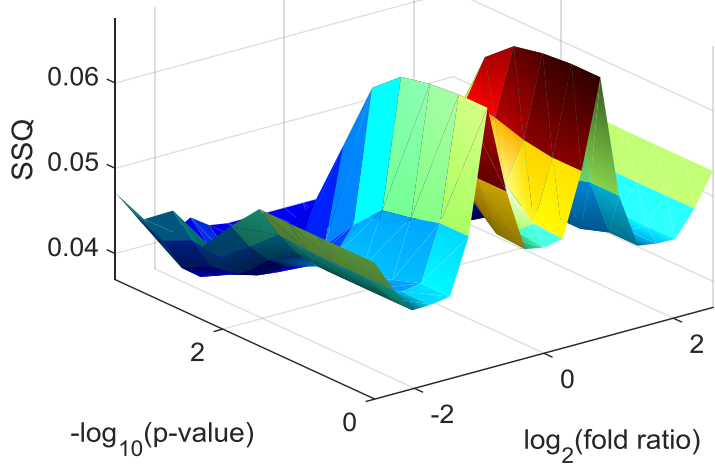
for the main effects in the simulated data set. Factors 1, 3 and 5 contained peaks affected by the factors, while the other factors have no effect on any of the peaks. 

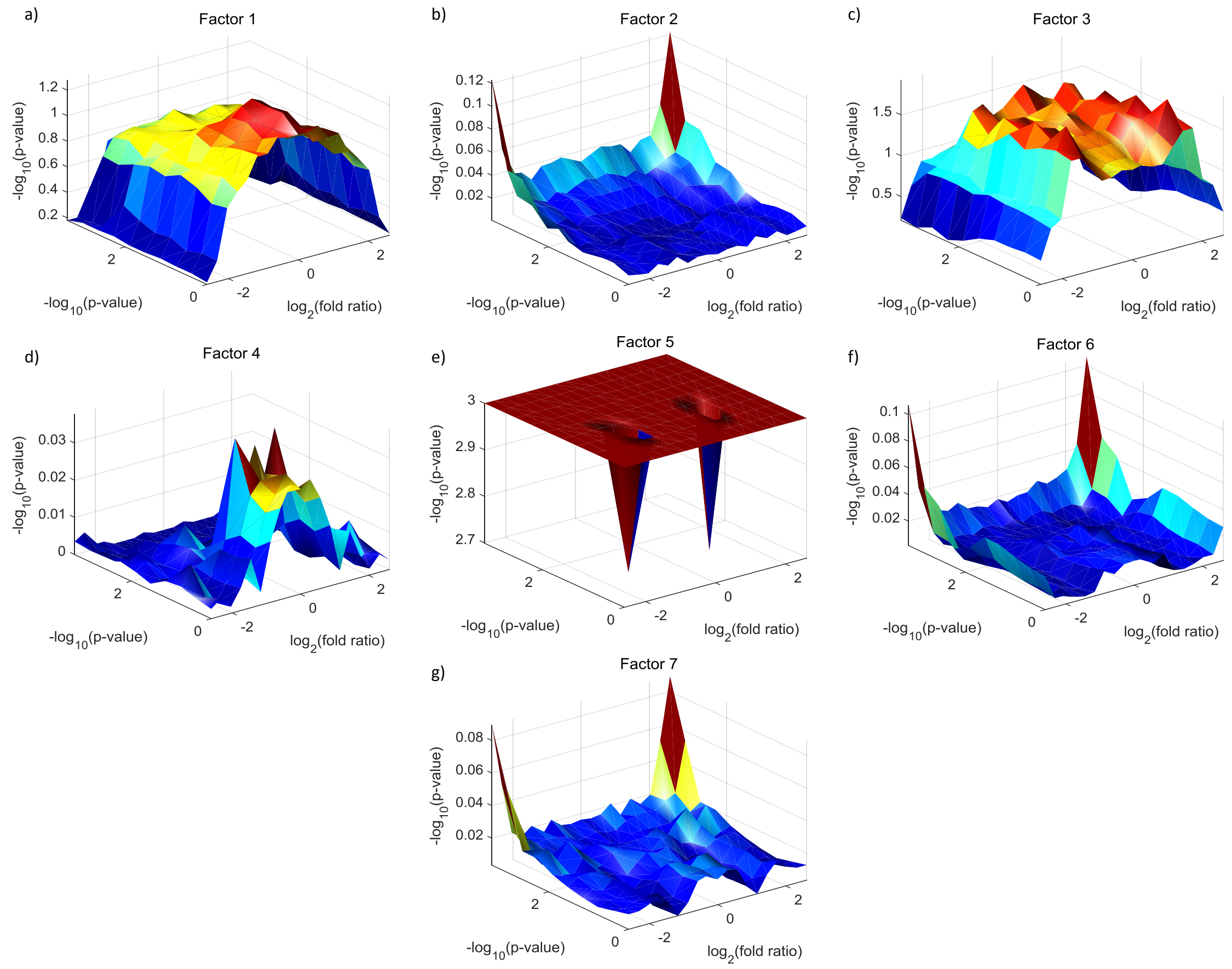

Figure S3. Surface plots showing the factor's ASCA variance significance (SSQ, z axis) as a function of $\log _{2}$ (fold change) and -log 10 ( $p$-value) $t$-test significance thresholds for the main effects in the simulated data set. Factors 1,3 and 5 contained peaks affected by the factors, while the other factors have no effect on any of the peaks. SSQ significance were set to 0.001 as lowest value that occurred in permutation test. 

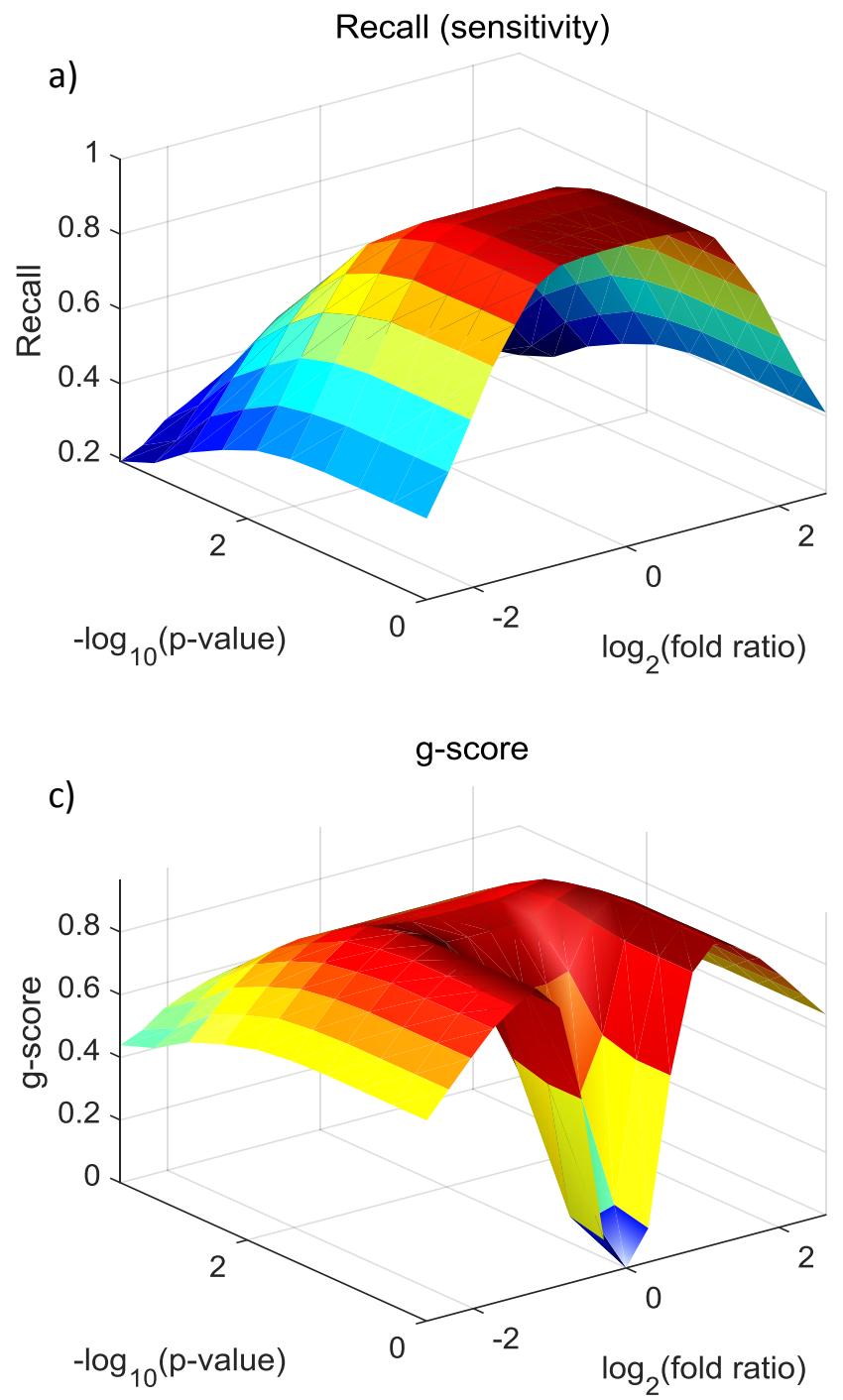

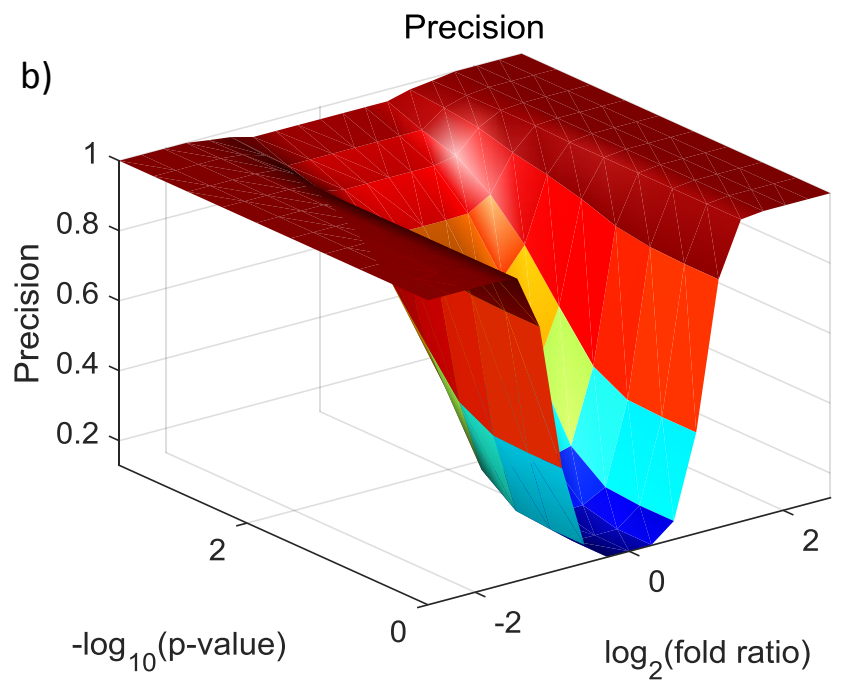

f-score

d)

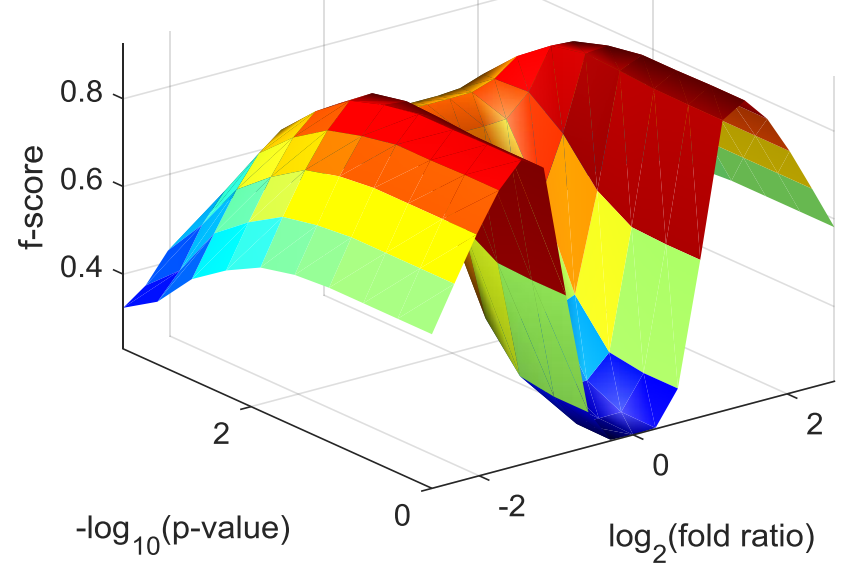

e) $\quad \log _{10}$ of number of selected variables

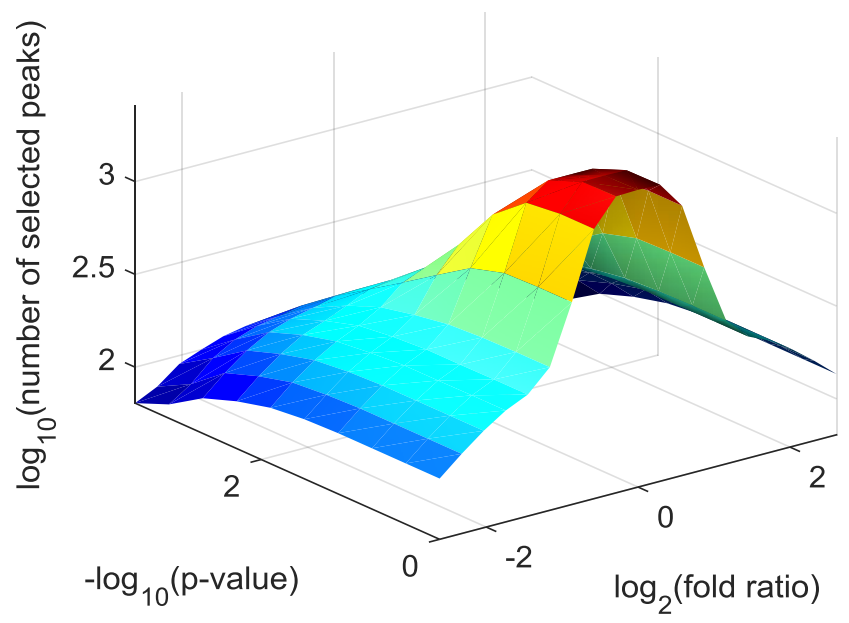

Figure S4. Surface plots showing the recall (a), precision (b), g-score (c) f-score (d) and $\log _{10}$ number of selected variables (e) as a function of $\log _{2}$ (fold change) and $-\log _{10}(p$-value) $t$-test significance thresholds for a simulated data matrix. 


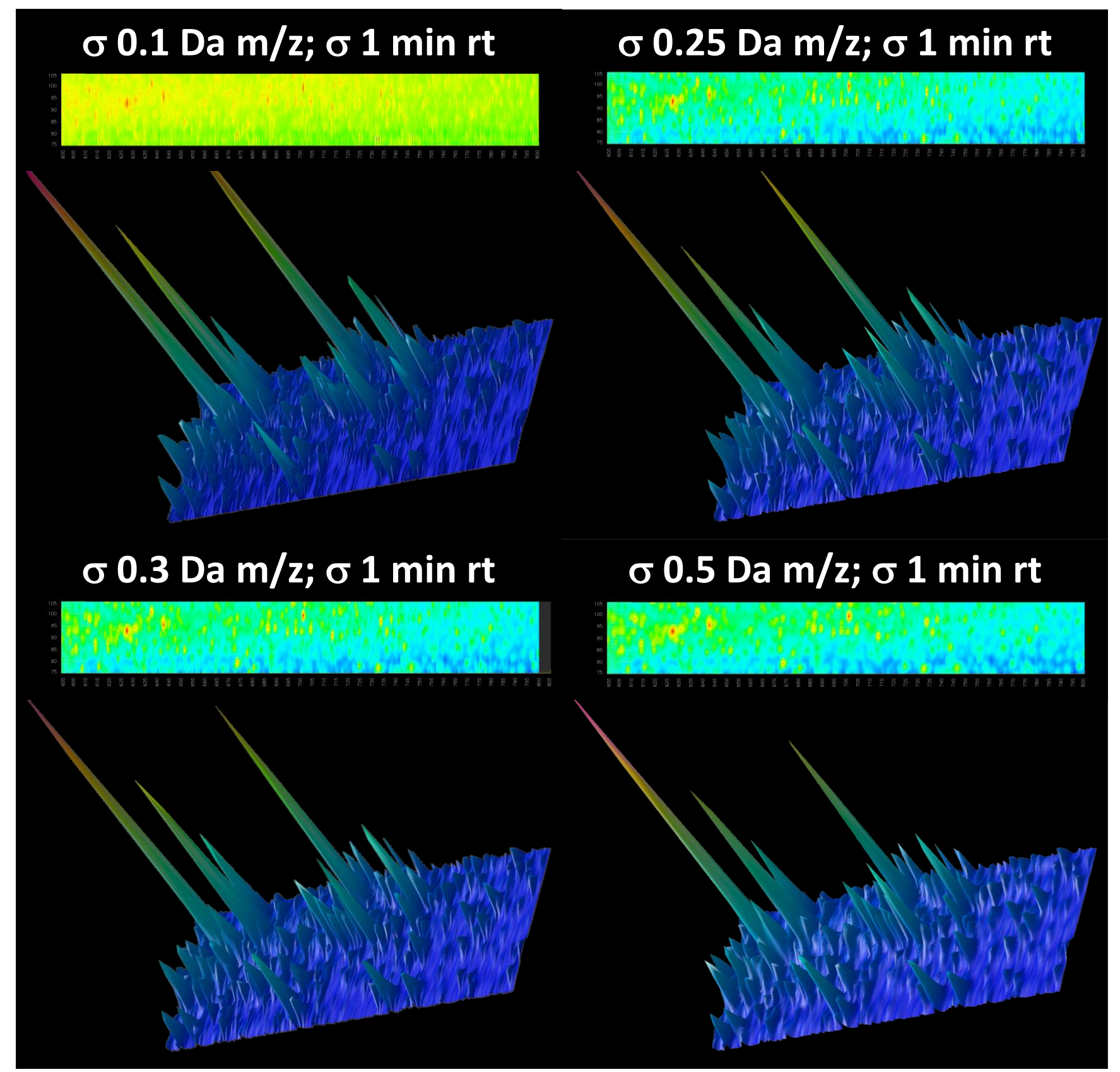

Figure S5. Optimization of the smoothing parameter for optimal quantitative pre-processing using the TAPP pipeline. The effect of parameters $\sigma_{\mathrm{rt}}$ and $\sigma_{\mathrm{m} / \mathrm{z}}$ (half the peak width at the inflection point of the Gaussian distribution) of the 2-dimensional Gaussian smoothing procedure on the noise content of single-stage MS data in Grid module of TAPP pipeline. The value of $\sigma$ in the retention time dimension $\left(\sigma_{\mathrm{rt}}\right)$ was 1 minute and the value of $\sigma$ in the $\mathrm{m} / \mathrm{z}$ dimension $\left(\sigma_{\mathrm{m} / \mathrm{z}}\right)$ was varied with $0.1,0.25,0.3$ and $0.5 \mathrm{Da}$. The $0.3 \mathrm{Da} \sigma_{\mathrm{m} / \mathrm{z}}$ provided the optimal settings for peak detection, without missing peaks (too much smoothing) or peak splitting (too much noise). These settings smoothed out isotopic clusters of one peptide with one charge state resulting in one Gaussian peak in the retention time, $\mathrm{m} / \mathrm{z}$ and ion count space. 

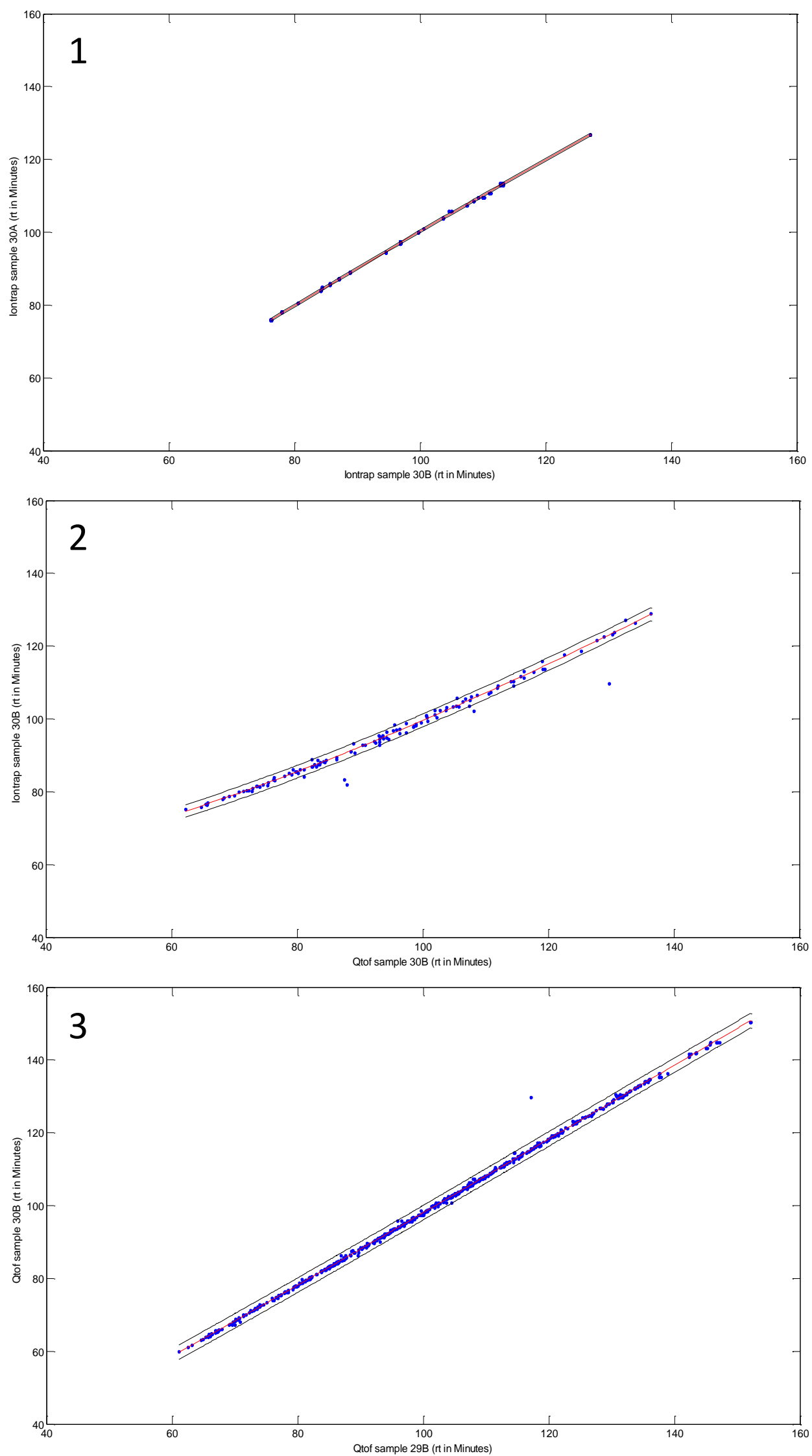

Figure S6. Scatterplot of identic MS/MS identifications in two chromatograms acquired with 1) ion trap (samples 30A and 30B), 2) ion trap and QTOF (sample 30B) and 3) QTOF (sample 30 B). The black lines show the values of $\mathrm{D}_{\max }(0.50,1.67$ and 2.00 respectively), while the red lines correspond to the main retention time correspondence trend ${ }^{6}$. 


\section{Same sample, different instrument}

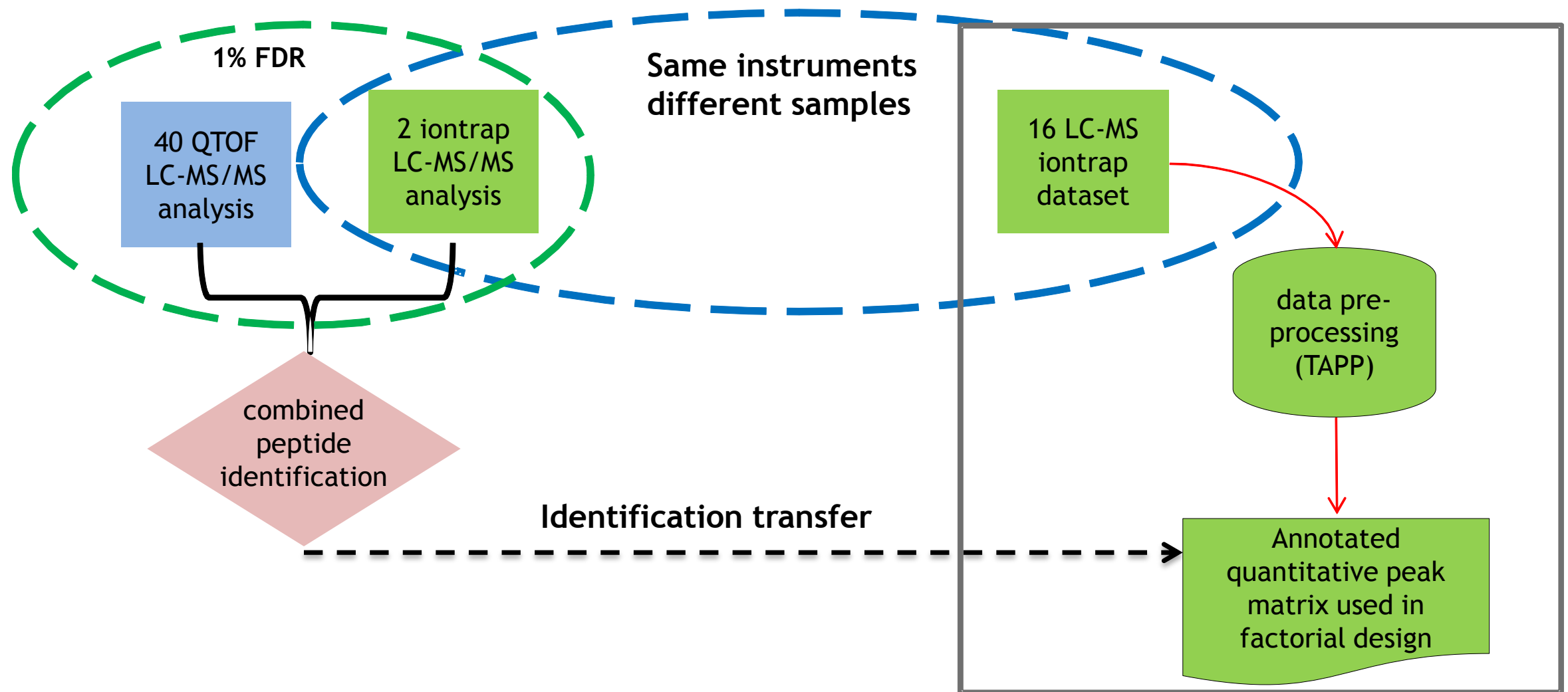

Figure S7. Main steps of the peptide identification transfer to annotate the quantitative peak matrix obtained from 16 LC-MS iontrap dataset acquired to study the effect of pre-analytical factor on depleted human serum peptide profile in experimental design study. 2 iontrap and 40 QTOF LC-MS/MS files were subjected to spectrum peptide match identification using PEAKS database search tool. These datasets were combined by using the retention time correspondence function obtained with retention time of identic MS/MS spectra in the two datasets aligning the identification of the 40 QTOF files to the aligned retention time domain of the 2 iontrap LC-MS/MS data. The 2 iontrap data were aligned to the best reference chromatogram of the 16 iontrap LC-MS chromatograms which allowed the transfer of combined identifications from 40 QTOF and 2 iontrap LC-MS/MS files by finding the highest peaks within retention window of $3.5 \mathrm{~min}$ and $0.85 \mathrm{~m} / \mathrm{z}$. 


\begin{tabular}{|l|l|l|}
\hline Truth by Methods & Selected peaks & Not selected peaks \\
\hline Affected peaks & True Positive (tp) & False Negative (fn) \\
\hline Not affected peaks & False Positive (fp) & True Negative (tn) \\
\hline
\end{tabular}

Table S1. Confusion table. The columns correspond to features as predicted by a given method, while the rows correspond to the actual class of the features. Adapted from Christin et al. ${ }^{9}$

\begin{tabular}{|l|l|}
\hline Measure & Equation \\
\hline $\begin{array}{l}\text { Sensitivity }=\text { Recall }=\text { True Positive Rate } \\
(\mathrm{TPR})\end{array}$ & $\frac{t p}{t p+f n}$ \\
\hline Precision & $\frac{t p}{t p+f p}$ \\
\hline Specificity = True Negative Rate $(\mathrm{TNR})$ & $\frac{t n}{t n+f p}$ \\
\hline Geometric Mean Accuracy $(g$-score $)$ & $\frac{\sqrt{T P R \cdot T N R}}{\left(\beta^{2}+1\right) \cdot \text { precision } \cdot \text { recall }}$ \\
\hline$f$-score & $\frac{\beta^{2} \cdot \text { precision }+ \text { recall }}{}$ \\
\hline
\end{tabular}

Table S2. Definition of the scores that were used to compare the performance of different feature selection methods. In this manuscript the value of $\beta$ in $f$-score calculation is 1 . Adapted from Christin et al. ${ }^{9}$ 


\begin{tabular}{lcll}
\hline \multicolumn{1}{c}{ Factor } & Peak rank & \multicolumn{1}{c}{ Peptide sequence } & \multicolumn{1}{c}{ Protein name } \\
\hline Heamolysis & 1 & VADALTNAVAHVDDMPNALSALSDLHAHK & Hemoglobin subunit alpha (P69905) \\
Heamolysis & 2 & FFESFGDLSTPDAVMGNPK & Hemoglobin subunit beta (P68871) \\
Heamolysis & 3 & VLGAFSDGLAHLDNLK & Hemoglobin subunit beta (P68871) \\
Heamolysis & 4 & VADALTNAVAHVDDMPNALSALSDLHAHK & Hemoglobin subunit alpha (P69905) \\
Heamolysis & 5 & VGFYESDVMGR & Alpha-2-macroglobulin (P01023) \\
Heamolysis & 6 & AIGYLNTGYQR & Alpha-2-macroglobulin (P01023) \\
Heamolysis & 7 & HVIILMTDGLHNM(Ox)GGDPITVIDEIR & Complement factor B precursor (P00751) \\
Heamolysis & 9 & FVTWIEGVM(Ox)R & Plasminogen (P00747) \\
Heamolysis & 10 & FFESFGDLSTPDAVMGNPK & Hemoglobin subunit beta (P68871) \\
Trypsin digestion & 1 & KFPSGTFEQVSQLVK & Vitamin D-binding protein (P02774) \\
Trypsin digestion & 4 & EQLGPVTQEF & Apolipoprotein A-I (P02647) \\
Trypsin digestion & 6 & AEAESLYQSK & Keratin, type II cytoskeletal 1 (P04264) \\
Trypsin digestion & 7 & FVELTMPYSVIR & Alpha-2-macroglobulin (P01023) \\
Trypsin digestion & 8 & PSLVPASAENVNK & Inter-alpha-trypsin inhibitor heavy chain \\
Trypsin digestion & 10 & ILTVPGHLDEM(Ox)QLDIQAR & H4 (Q14624) \\
Stopping Trypsin & 3 & VVNNSPQPQNVVFDVQIPK & Complement C4-A and B (P0C0L5) \\
Stopping Trypsin & 7 & YFKPGMPFDLMV & Inter-alpha-trypsin inhibitor heavy chain \\
Stopping Trypsin & 9 & DFVQPPTK & H2 (P19823) \\
\hline & & & Complement C3 (P01024) \\
& & Kininogen-1 (P01042) \\
\hline
\end{tabular}

Table S3. Peptide sequences and protein names of the most discriminating, annotated peaks for the 3 factors that affect depleted human serum peptide profiles. The peak rank reflects the discriminating rank of the peak according to the average absolute ASCA loadings obtained with 100 repetitions of the ASCA analysis as displayed in the bar diagrams of Figure 3. The protein name reflect the occurrence of the peptides in SwissProt entries.

\section{Reference}

(1) Holman, J. D.; Tabb, D. L.; Mallick, P. Current protocols in bioinformatics / editoral board, Andreas D. Baxevanis ... [et al.] 2014, 46, 1324 11-19.

(2) Kessner, D.; Chambers, M.; Burke, R.; Agus, D.; Mallick, P. Bioinformatics 2008, 24, 2534-2536.

(3) Suits, F.; Hoekman, B.; Rosenling, T.; Bischoff, R.; Horvatovich, P. Analytical Chemistry 2011, 83, 77867794.

(4) Ahmad, I.; Suits, F.; Hoekman, B.; Swertz, M. A.; Byelas, H.; Dijkstra, M.; Hooft, R.; Katsubo, D.; van Breukelen, B.; Bischoff, R.; Horvatovich, P. Bioinformatics (Oxford, England) 2011, 27, 1176-1178.

(5) Zhang, J.; Xin, L.; Shan, B.; Chen, W.; Xie, M.; Yuen, D.; Zhang, W.; Zhang, Z.; Lajoie, G. A.; Ma, B. Molecular \& cellular proteomics : MCP 2012, 11, M111 010587.

(6) Zwanenburg, G.; Hoefsloot, H. C. J.; Westerhuis, J. A.; Jansen, J. J.; Smilde, A. K. Journal of Chemometrics 2011, 25, 561-567.

(7) Mitra, V.; Smilde, A.; Hoefsloot, H.; Suits, F.; Bischoff, R.; Horvatovich, P. Journal of chromatography. A 2014, 1373, 61-72.

(8) Cox, J.; Mann, M. Nature biotechnology 2008, 26, 1367-1372.

(9) Christin, C.; Hoefsloot, H. C.; Smilde, A. K.; Hoekman, B.; Suits, F.; Bischoff, R.; Horvatovich, P. Mol Cell Proteomics 2013, 12, 263-276. 